International Journal of Current Microbiology and Applied Sciences

ISSN: 2319-7706 Volume 8 Number 02 (2019)

Journal homepage: http://www.ijcmas.com

Original Research Article

https://doi.org/10.20546/ijcmas.2019.802.221

\title{
Effect of Storage Conditions on Seed Longevity of Onion (Allium cepa L.)
}

\author{
C. Geetanjali ${ }^{*}$, I.M. Sangeeta ${ }^{1}$, S.M. Prashant ${ }^{1}$, Basavegowda ${ }^{1}$ and R.V. Beladhadi ${ }^{2}$ \\ ${ }^{1}$ Department of Seed Science and Technology, ${ }^{2}$ Department of Soil Science, University of \\ Agricultural Sciences, Raichur-584 104, Karnataka, India
}

*Corresponding author

\section{A B S T R A C T}

\section{Keywords}

Onion, storage

conditions,

Packaging

materials, Seed

quality,

Commercial cold

storage

Article Info

Accepted:

15 January 2019

Available Online:

10 February 2019
An experiment was conducted to know the effect of storage conditions on seed quality of onion cv. Arka kalyan. The experiment consisted of three storage conditions (ambient condition, conditioned cold storage $\left(18-20^{\circ} \mathrm{C}\right.$, $45-50 \% \mathrm{RH})$, commercial cold storage $\left(5-7{ }^{\circ} \mathrm{C}, 65 \% \mathrm{RH}\right)$. The results revealed that, seeds stored in commercial cold storage $\left(5-7^{\circ} \mathrm{C}+65 \% \mathrm{RH}\right)$ recorded significantly higher seed germination, speed of germination, test weight, root length, shoot length, seedling dry weight, vigour index, lower moisture content and electrical conductivity after 10 months of storage. Hence commercial cold storage was found to be best for maintaining seed quality parameter.

\section{Introduction}

Onion (Allium cepa L.) is one of the commercially important spice vegetable crops grown all over the world. It is a member of Amaryllidaceae $\quad(2 n=16 \quad$ chromosome $)$, belongs to herbaceous monocot bulbous plant with annual bulb production and biennial seed production. Seed is the foundation of agriculture for enhancing crop production, but the availability of quality seed is the main constraint to crop production in developing country like India. Onion seed production takes longer time as compared with other bulb and spice crops. There are many factors which affect the seed quality and growth potential attributes of many crops, among them, use of quality seeds is the most important one. The quality of the seed is highest when it completes structural and functional development on plant itself. Thereafter, it deteriorates irreparably and irreversibly at varying rates (Delouche and Baskin 1973). 
Onion is a poor storer and hence seeds losses its viability very quickly. Onion is an important vegetable crop and good seed is a basic input in vegetable production. Its seed are short lived under ambient conditions (Doijode, 1987). Since, seed is a living entity and is subjected to various environmental stresses which affect the quality. Regular fluctuation in temperature, moisture content makes the storage of seeds troublesome (Suma et al., 2013). Under unfavourable storage condition such as high temperature and relative humidity the variation in seed germination rate can be high (Sisman, 2005). In storage, the declination of germinability with high moisture content is related to the hygroscopic nature of seeds, especially under worm temperatures, which in turn is associated with the relative humidity of surrounding air at ambient condition. After ten months of storage period, maximum decrease in all the seed quality parameters with higher moisture content was noticed in onion seeds. In this view the present investigation entitled "effect of storage conditions on seed longevity of onion (Allium сера L.)" was undertaken.

\section{Materials and Methods}

The present investigation was carried out in laboratory of the Department of Seed Science and Technology, College of Agriculture, University of Agricultural Science, Raichur. Fresh seeds of onion variety Arka Kalyan having initial germination of 95 per cent was obtained from Agricultural Research Station, Hagari, Bellary District Karnataka. The experiment consisted of three storage conditions viz., $\mathrm{C}_{1}$ : Ambient condition $\mathrm{C}_{2}$ : Conditioned cold storage $\left(18-20^{\circ} \mathrm{C}, 45-50 \%\right.$ $\mathrm{RH})$ and $\mathrm{C}_{3}$ : Commercial cold storage $\left(5-7^{\circ} \mathrm{C}\right.$, $65 \% \mathrm{RH})$. All the seeds were treated with Carbendazim $2 \mathrm{~g} / \mathrm{kg}$ and stored for ten months. The experiment was carried out using Completely Randomized Design (Factorial) repeated four times. Seeds were packed in three different packing materials and stored in three different storage conditions. Observations were recorded at monthly interval on germination (\%), speed of germination, root length $(\mathrm{cm})$, shoot length $(\mathrm{cm})$, seedling dry weight $(\mathrm{mg})$, seed vigour index, seed moisture content (\%) and biochemical observation viz., dehydrogenase activity, alpha amylase enzyme activity and electrical conductivity were recorded at bimonthly intervals. Germination test was carried out using paper towel technique as per the procedure given by ISTA (1999). Germinated seeds were counted on $12^{\text {th }}$ day and 10 germinated seedlings were selected from each replication of the treatment for calculating the seedling vigour index. The seedling vigour index (length and mass) was calculated by using the formula given by Abdul-Baki and Anderson, 1973. The shoot and root length of each of the 10 seedlings were measured in centimeters. Seedling dry weight (mg) was measured for all the germinated seedlings after oven drying. Electrical conductivity of the seed leachate was measured in the digital conductivity bridge (WENSAR) with a cell constant 1.0 and the mean values were expressed in decisiemens per meter $\left(\mathrm{dSm}^{-1}\right)$ (Milosevis et $a l ., 2010)$. The OD value of dehydrogenase enzyme activity was obtained as reported by Kittock and Law, (1968). The $\alpha$-amylase enzyme activity was analysed as per the method suggested by Simpson and Naylor (1962). The data were statistically analyzed as per the method of Cochran and Cox (1957) for Completely Randomized Design (Factorial).

\section{Results and Discussion}

Results of storage conditions on moisture content of Arka kalyan represented in Table 1. The temperature maintained in commercial cold storage was $5-7^{\circ} \mathrm{C}$ and relative humidity 
was 65 per cent. The onion seeds packed in pervious packaging materials and stored in commercial cold storage gained moisture content and this is evidenced by rise in moisture content from 6.00 to 7.68 per cent at the end of storage period. This was due to prevalence of higher $\mathrm{RH}$ or vapour pressure and low temperature in cold storage. The seeds stored in ambient condition also gained moisture content and it is evidenced by rise in moisture content to 7.56 per cent from 6.00 per cent due to prevalence of higher vapour pressure. The relative humidity under ambient condition varied from 87 to 62 per cent during storage period. The vapour pressure in commercial cold storage was more hence moisture content in seeds was also more (7.68 $\%$ ) compared to seeds stored in ambient condition. These results are in accordance with findings of Harrington (1960) who has reported the higher moisture content of seed controlled by relative humidity and temperature in the storage environment. Similar findings were also been recorded by Devanal et al., (2012) in sunflower and Basavegowda et al., (2013) in chickpea, $\mathrm{Na}$ et al., (2013) in sesame and Meena et al., (2017) in cotton and groundnut.

Results of storage conditions on test weight of Arka kalyan represented in Table 2. Higher test weight of seeds was noticed in seeds stored under ambient condition which was due to the moisture absorbance by the seeds due to humidity. Lowest test weight of seeds was noticed in seeds stored in cold condition which is due lower humidity levels in the storage conditions. In present experiment, the highest test weight $(3.53 \mathrm{~g})$ was recorded in ambient condition $\left(\mathrm{C}_{1}\right)$ which was followed by conditioned cold storage $\left(\mathrm{C}_{2}\right)(3.52 \mathrm{~g})$ and lowest was recorded in commercial cold storage $\left(\mathrm{C}_{3}\right)(3.51 \mathrm{~g})$ after one month of storage. This was influenced by many different factors such as seed potential, humidity and storage condition and seed quality. At the end of storage period $\mathrm{C}_{1}$ recorded significantly higher test weight (3.08 g) which was on par with $\mathrm{C}_{2}(3.07 \mathrm{~g})$ and significantly lowest was recorded in commercial cold storage $\mathrm{C}_{3}(2.97 \mathrm{~g})$. The results are in accordance with the earlier findings of Malcolm et al., (2003) in peach, Akbari et al., (2004) in soybean and Mashtati et al., (2008) in wheat.

Germination is the most important function of a seed as an indicator of its viability and worth as seed (Table 2 and 3). The results revealed that after one month of storage, highest germination and speed of germination (96.40 \% and 34.90 respectively) were recorded in commercial cold storage $\left(\mathrm{C}_{3}\right)$ followed by conditioned cold storage $\left(\mathrm{C}_{2}\right)$ (95.75\% and 34.61 respectively) but seeds stored in ambient condition $\left(\mathrm{C}_{1}\right)$ recorded lower germination and speed of germination (95.02 \% and 34.21 respectively). The increased germination under controlled condition may be attributed to low temperature decrease the rate of deterioration of seeds but it was high under ambient condition. There was gradual reduction in the germination percentage from one to ten months of storage in all the conditions, however at the end of tenth month of storage, the seeds stored at commercial cold storage $\left(\mathrm{C}_{3}\right)$ recorded germination above minimum seed certification standards and highest speed of germination $(85.54 \%$ and 25.78 respectively) followed by conditioned cold storage $\left(\mathrm{C}_{2}\right)$ (78.91\% and 25.06 respectively) and significantly lowest values were observed in ambient condition $\left(\mathrm{C}_{1}\right)(62.12 \%$ and 15.80 respectively). Higher seed quality parameter in lower temperature might be attributed to reduced metabolic activity like reduced lipid peroxidation resulted in enhanced antioxidant activity which protects seeds from deterioration. Alhamdan et al., (2011) reported that seeds of onion stored at $5^{\circ} \mathrm{C}$ had the highest seed germination percentage. 
Results obtained under the present study are supported by the findings of Hanna et al., (2013) and Demir et al., (2016) in onion.

Results of storage conditions on root length and shoot length of Arka kalyan represented in Table 4 and 5. In the present study at the end of first month of storage period, the highest root length $(7.98 \mathrm{~cm})$ and shoot length $(7.57 \mathrm{~cm})$ were recorded in commercial cold storage $\left(\mathrm{C}_{3}\right)$ which was followed by conditioned cold storage $\left(\mathrm{C}_{2}\right)(7.97 \mathrm{~cm}$ and $7.56 \mathrm{~cm}$ respectively) whereas, the lowest values were recorded in ambient condition $\left(\mathrm{C}_{1}\right)(7.96 \mathrm{~cm}$ and $7.55 \mathrm{~cm}$ respectively). Seeds preserved in cold storage recorded higher root length and shoot length as compared to ambient condition which may be due to lower respiration rate and metabolic activity at lower temperature, hence the higher seedling length is an indication of maintenance of vigour in the seeds preserved in cold storage. At the end of tenth month of storage period, seed stored at commercial cold storage $\left(\mathrm{C}_{3}\right)$ recorded significantly maximum root length $(7.03 \mathrm{~cm})$ and shoot length $(6.88$ $\mathrm{cm})$ which was followed by conditioned cold storage $\left(\mathrm{C}_{2}\right) \quad(6.65 \mathrm{~cm}$ and $6.42 \mathrm{~cm}$ respectively) and lowest was recorded in ambient condition $\left(\mathrm{C}_{1}\right)(5.70 \mathrm{~cm}$ and $5.30 \mathrm{~cm}$ respectively). Similar findings reported by Balesevic-Tubic et al., (2010) in oilseed crops, Khalequzzaman et al., (2012) in frenchbean, Basavegowda et al., (2013) in chickpea, Venge et al., (2016) in soybean and Patel et al., (2017) in onion.

Results of storage conditions on seedling dry weight of Arka kalyan represented in Table 6. The highest seedling dry weight was recorded in commercial cold storage $\left(\mathrm{C}_{3}\right)(22.16 \mathrm{mg})$ which was followed by conditioned cold storage $\left(\mathrm{C}_{2}\right)(22.13 \mathrm{mg})$ whereas minimum seedling dry weight was noticed $(21.98 \mathrm{mg})$ in ambient condition $\left(\mathrm{C}_{1}\right)$. Reduction in seedling dry weight might be due to shorter root and shoot length registered in our study which had a direct correlation with dry weight. Following the same trend, the seeds stored in commercial cold storage $\left(\mathrm{C}_{3}\right)$ recorded significantly highest seedling dry weight $(15.01 \mathrm{mg})$ which was followed by conditioned cold storage $\mathrm{C}_{2}(12.98 \mathrm{mg})$ and significantly lowest seedling dry weight was recorded in ambient condition $\mathrm{C}_{1}(10.36 \mathrm{mg})$. Similar findings were given by Kandil et al., (2013) in soybean, Demir et al., (2016) in lettuce and Kavitha et al., (2017) in sesame.

Table.1 Effect of storage conditions on moisture content (\%) of onion seed during storage

\begin{tabular}{|c|c|c|c|c|c|c|c|c|c|c|}
\hline \multirow{2}{*}{ Treatment } & \multicolumn{10}{|c|}{ Storage period in months } \\
\cline { 2 - 13 } & $\mathbf{1}$ & $\mathbf{2}$ & $\mathbf{3}$ & $\mathbf{4}$ & $\mathbf{5}$ & $\mathbf{6}$ & $\mathbf{7}$ & $\mathbf{8}$ & $\mathbf{9}$ & $\mathbf{1 0}$ \\
\hline $\mathbf{C}_{\mathbf{1}}$ & 6.99 & 7.23 & 7.37 & 7.82 & 8.11 & 8.32 & 8.13 & 7.88 & 7.64 & 7.56 \\
\hline $\mathbf{C}_{\mathbf{2}}$ & 6.84 & 6.85 & 6.86 & 6.90 & 7.04 & 7.14 & 7.22 & 7.37 & 7.53 & 7.58 \\
\hline $\mathbf{C}_{\mathbf{3}}$ & 6.94 & 7.04 & 7.08 & 7.16 & 7.23 & 7.32 & 7.40 & 7.48 & 7.59 & 7.68 \\
\hline Mean & 6.92 & 7.04 & 7.10 & 7.29 & 7.46 & 7.59 & 7.58 & 7.57 & 7.59 & 7.61 \\
\hline SEm \pm & 0.19 & 0.14 & 0.06 & 0.06 & 0.08 & 0.04 & 0.08 & 0.07 & 0.04 & 0.07 \\
\hline CD at 1 \% & NS & NS & 0.24 & 0.22 & 0.31 & 0.15 & 0.31 & 0.28 & 0.16 & 0.27 \\
\hline
\end{tabular}

NS - Non significant; Storage conditions $(\mathrm{C}) ; \mathrm{C}_{1}-$ Ambient storage condition; $\mathrm{C}_{2}$ - Conditioned cold storage $\left(18-20{ }^{\circ} \mathrm{C}, 45-50 \% \mathrm{RH}\right) ; \mathrm{C}_{3}-\mathrm{Commercial}$ cold storage condition; (5 to $7^{\circ} 65 \% \mathrm{RH}$ ) 
Table.2 Effect of storage conditions on germination (\%) of onion seed during storage

\begin{tabular}{|c|c|c|c|c|c|c|c|c|c|c|}
\hline \multirow[t]{2}{*}{ Treatment } & \multicolumn{10}{|c|}{ Storage period in months } \\
\hline & 1 & 2 & 3 & 4 & 5 & 6 & 7 & 8 & 9 & 10 \\
\hline $\mathbf{C}_{1}$ & $\begin{array}{c}95.02 \\
(77.13)\end{array}$ & $\begin{array}{c}94.03 \\
(75.86)\end{array}$ & $\begin{array}{c}91.17 \\
(72.72)\end{array}$ & $\begin{array}{c}87.45 \\
(69.27)\end{array}$ & $\begin{array}{c}84.52 \\
(66.89)\end{array}$ & $\begin{array}{c}80.34 \\
(63.76)\end{array}$ & $\begin{array}{c}75.37 \\
(60.35)\end{array}$ & $\begin{array}{c}71.07 \\
(57.57)\end{array}$ & $\begin{array}{c}66.85 \\
(54.97)\end{array}$ & $\begin{array}{c}62.12 \\
(52.23)\end{array}$ \\
\hline $\mathrm{C}_{2}$ & $\begin{array}{c}95.75 \\
(78.11)\end{array}$ & $\begin{array}{c}94.55 \\
(76.51)\end{array}$ & $\begin{array}{c}92.66 \\
(74.30)\end{array}$ & $\begin{array}{c}90.52 \\
(72.08)\end{array}$ & $\begin{array}{c}89.10 \\
(70.74)\end{array}$ & $\begin{array}{c}87.47 \\
(69.32)\end{array}$ & $\begin{array}{c}84.65 \\
(66.97)\end{array}$ & $\begin{array}{c}82.76 \\
(65.51)\end{array}$ & $\begin{array}{c}81.10 \\
(64.32)\end{array}$ & $\begin{array}{c}78.91 \\
(62.70)\end{array}$ \\
\hline $\mathbf{C}_{3}$ & $\begin{array}{c}96.40 \\
(79.75)\end{array}$ & $\begin{array}{c}95.58 \\
(78.40)\end{array}$ & $\begin{array}{c}94.42 \\
(77.10)\end{array}$ & $\begin{array}{c}93.46 \\
(75.46)\end{array}$ & $\begin{array}{c}92.19 \\
(74.11)\end{array}$ & $\begin{array}{c}90.68 \\
(72.55)\end{array}$ & $\begin{array}{c}89.24 \\
(71.23)\end{array}$ & $\begin{array}{c}87.89 \\
(70.04)\end{array}$ & $\begin{array}{c}86.81 \\
(69.16)\end{array}$ & $\begin{array}{c}85.54 \\
(67.83) \\
\end{array}$ \\
\hline Mean & $\begin{array}{c}95.72 \\
(78.33)\end{array}$ & $\begin{array}{c}94.72 \\
(76.92)\end{array}$ & $\begin{array}{c}92.75 \\
(74.71)\end{array}$ & $\begin{array}{c}90.48 \\
(72.27)\end{array}$ & $\begin{array}{c}88.60 \\
(70.58)\end{array}$ & $\begin{array}{c}86.16 \\
(68.55)\end{array}$ & $\begin{array}{c}83.08 \\
(66.18)\end{array}$ & $\begin{array}{c}80.57 \\
(64.37)\end{array}$ & $\begin{array}{c}78.25 \\
(62.81)\end{array}$ & $\begin{array}{c}75.52 \\
(60.92)\end{array}$ \\
\hline SEm \pm & 0.39 & 0.40 & 0.36 & 0.32 & 0.28 & 0.20 & 0.24 & 0.25 & 0.27 & 0.29 \\
\hline CD at $1 \%$ & NS & NS & 1.26 & 1.12 & 0.98 & 0.70 & 0.84 & 0.87 & 0.94 & 1.01 \\
\hline
\end{tabular}

NS - Non significant; Storage conditions $(\mathrm{C}) ; \mathrm{C}_{1}-$ Ambient storage condition; $\mathrm{C}_{2}$ - Conditioned cold storage

$\left(18-20^{\circ} \mathrm{C}, 45-50 \% \mathrm{RH}\right) ; \mathrm{C}_{3}-$ Commercial cold storage condition; (5 to $\left.7^{\circ} 65 \% \mathrm{RH}\right)$

Table.3 Effect of storage conditions on speed of germination of onion seed during storage

\begin{tabular}{|c|c|c|c|c|c|c|c|c|c|c|}
\hline \multirow{2}{*}{ Treatment } & \multicolumn{10}{|c|}{ Storage period in months } \\
\cline { 2 - 13 } & $\mathbf{1}$ & $\mathbf{2}$ & $\mathbf{3}$ & $\mathbf{4}$ & $\mathbf{5}$ & $\mathbf{6}$ & $\mathbf{7}$ & $\mathbf{8}$ & $\mathbf{9}$ & $\mathbf{1 0}$ \\
\hline $\mathbf{C}_{\mathbf{1}}$ & 34.21 & 33.67 & 32.10 & 31.27 & 29.91 & 28.07 & 25.01 & 21.71 & 18.70 & 15.80 \\
\hline $\mathbf{C}_{\mathbf{2}}$ & 34.61 & 34.07 & 33.77 & 33.31 & 32.22 & 30.77 & 29.15 & 27.95 & 26.47 & 25.06 \\
\hline $\mathbf{C}_{\mathbf{3}}$ & 34.90 & 34.43 & 34.12 & 33.79 & 32.84 & 31.62 & 30.50 & 29.11 & 27.60 & 25.78 \\
\hline Mean & 34.57 & 34.06 & 33.33 & 32.79 & 31.66 & 30.16 & 28.22 & 26.26 & 24.26 & 22.21 \\
\hline SEm \pm & 0.36 & 0.31 & 0.07 & 0.08 & 0.06 & 0.06 & 0.07 & 0.08 & 0.07 & 0.05 \\
\hline CD at 1 \% & NS & NS & 0.24 & 0.28 & 0.21 & 0.21 & 0.24 & 0.28 & 0.24 & 0.17 \\
\hline
\end{tabular}

NS - Non significant; Storage conditions (C); $\mathrm{C}_{1}-$ Ambient storage condition; $\mathrm{C}_{2}$ - Conditioned cold storage

$\left(18-20{ }^{\circ} \mathrm{C}, 45-50 \% \mathrm{RH}\right) ; \mathrm{C}_{3}-$ Commercial cold storage condition; $\left(5\right.$ to $\left.7{ }^{\circ} 65 \% \mathrm{RH}\right)$

Table.4 Effect of storage conditions on root length $(\mathrm{cm})$ of onion seed during storage

\begin{tabular}{|c|c|c|c|c|c|c|c|c|c|c|}
\hline \multirow[t]{2}{*}{ Treatment } & \multicolumn{10}{|c|}{ Storage period in months } \\
\hline & 1 & 2 & 3 & 4 & 5 & 6 & 7 & 8 & 9 & 10 \\
\hline $\mathbf{C}_{1}$ & 7.96 & 7.92 & 7.70 & 7.50 & 7.23 & 7.02 & 6.72 & 6.36 & 6.04 & 5.70 \\
\hline $\mathbf{C}_{2}$ & 7.97 & 7.93 & 7.86 & 7.74 & 7.63 & 7.46 & 7.31 & 7.09 & 6.87 & 6.65 \\
\hline $\mathbf{C}_{3}$ & 7.98 & 7.94 & 7.87 & 7.79 & 7.71 & 7.57 & 7.49 & 7.33 & 7.18 & 7.03 \\
\hline Mean & 7.97 & 7.93 & 7.81 & 7.68 & 7.52 & 7.35 & 7.17 & 6.93 & 6.70 & 6.46 \\
\hline SEm \pm & 0.068 & 0.024 & 0.005 & 0.005 & 0.007 & 0.010 & 0.006 & 0.006 & 0.007 & 0.006 \\
\hline CD at $1 \%$ & NS & NS & 0.017 & 0.017 & 0.024 & 0.035 & 0.021 & 0.021 & 0.024 & 0.020 \\
\hline
\end{tabular}

NS - Non significant; Storage conditions (C); $\mathrm{C}_{1}-$ Ambient storage condition; $\mathrm{C}_{2}$ - Conditioned cold storage

$\left(18-20{ }^{\circ} \mathrm{C}, 45-50 \% \mathrm{RH}\right) ; \mathrm{C}_{3}-\mathrm{Commercial}$ cold storage condition; $\left(5\right.$ to $\left.7{ }^{\circ} 65 \% \mathrm{RH}\right)$

Table.5 Effect of storage conditions on shoot length $(\mathrm{cm})$ of onion seed during storage

\begin{tabular}{|c|c|c|c|c|c|c|c|c|c|c|}
\hline \multirow{2}{*}{ Treatment } & \multicolumn{10}{|c|}{ Storage period in months } \\
\cline { 2 - 13 } & $\mathbf{1}$ & $\mathbf{2}$ & $\mathbf{3}$ & $\mathbf{4}$ & $\mathbf{5}$ & $\mathbf{6}$ & $\mathbf{7}$ & $\mathbf{8}$ & $\mathbf{9}$ & $\mathbf{1 0}$ \\
\hline $\mathbf{C}_{\mathbf{1}}$ & 7.55 & 7.54 & 7.51 & 7.42 & 7.34 & 7.16 & 6.87 & 6.40 & 5.89 & 5.30 \\
\hline $\mathbf{C}_{\mathbf{2}}$ & 7.56 & 7.55 & 7.52 & 7.47 & 7.41 & 7.31 & 7.14 & 6.91 & 6.67 & 6.42 \\
\hline $\mathbf{C}_{\mathbf{3}}$ & 7.57 & 7.55 & 7.54 & 7.50 & 7.44 & 7.36 & 7.26 & 7.12 & 7.00 & 6.88 \\
\hline Mean & 7.56 & 7.54 & 7.52 & 7.46 & 7.40 & 7.27 & 7.09 & 6.81 & 6.52 & 6.20 \\
\hline SEm \pm & 0.017 & 0.014 & 0.004 & 0.004 & 0.011 & 0.005 & 0.004 & 0.013 & 0.020 & 0.012 \\
\hline CD at 1 \% & NS & NS & 0.014 & 0.014 & 0.038 & 0.017 & 0.014 & 0.045 & 0.070 & 0.042 \\
\hline
\end{tabular}

NS - Non significant; Storage conditions (C); $\mathrm{C}_{1}-$ Ambient storage condition; $\mathrm{C}_{2}$ - Conditioned cold storage

$\left(18-20{ }^{\circ} \mathrm{C}, 45-50 \% \mathrm{RH}\right) ; \mathrm{C}_{3}-$ Commercial cold storage condition; $\left(5\right.$ to $\left.7{ }^{\circ} 65 \% \mathrm{RH}\right)$ 
Table.6 Effect of storage conditions on seedling dry weight $(\mathrm{mg})$ of onion seed during storage

\begin{tabular}{|c|c|c|c|c|c|c|c|c|c|c|}
\hline \multirow{2}{*}{ Treatment } & \multicolumn{10}{|c|}{ Storage period in months } \\
\cline { 2 - 13 } & $\mathbf{1}$ & $\mathbf{2}$ & $\mathbf{3}$ & $\mathbf{4}$ & $\mathbf{5}$ & $\mathbf{6}$ & $\mathbf{7}$ & $\mathbf{8}$ & $\mathbf{9}$ & $\mathbf{1 0}$ \\
\hline $\mathbf{C}_{\mathbf{1}}$ & 21.98 & 21.49 & 20.99 & 20.47 & 19.99 & 19.21 & 18.02 & 16.37 & 13.36 & 10.36 \\
\hline $\mathbf{C}_{\mathbf{2}}$ & 22.13 & 21.62 & 21.10 & 20.63 & 20.13 & 19.25 & 18.37 & 17.00 & 14.99 & 12.98 \\
\hline $\mathbf{C}_{\mathbf{3}}$ & 22.16 & 21.77 & 21.19 & 20.68 & 20.17 & 19.35 & 18.59 & 17.71 & 16.37 & 15.01 \\
\hline Mean & 22.09 & 21.62 & 21.09 & 20.59 & 20.10 & 19.27 & 18.32 & 17.03 & 14.91 & 12.78 \\
\hline SEm \pm & 0.36 & 0.41 & 0.01 & 0.01 & 0.02 & 0.02 & 0.01 & 0.02 & 0.02 & 0.02 \\
\hline CD at 1 \% & NS & NS & 0.03 & 0.04 & 0.06 & 0.07 & 0.03 & 0.06 & 0.07 & 0.06 \\
\hline
\end{tabular}

NS - Non significant; Storage conditions (C); $\mathrm{C}_{1}-$ Ambient storage condition; $\mathrm{C}_{2}$ - Conditioned cold storage $\left(18-20{ }^{\circ} \mathrm{C}, 45-50 \% \mathrm{RH}\right) ; \mathrm{C}_{3}-\mathrm{Commercial}$ cold storage condition; $\left(5\right.$ to $\left.7{ }^{\circ} 65 \% \mathrm{RH}\right)$

Table.7 Effect of storage conditions on seedling vigour index of onion seed during storage

\begin{tabular}{|c|c|c|c|c|c|c|c|c|c|c|}
\hline \multirow[t]{2}{*}{ Treatment } & \multicolumn{10}{|c|}{ Storage period in months } \\
\hline & 1 & 2 & 3 & 4 & 5 & 6 & 7 & 8 & 9 & 10 \\
\hline $\mathbf{C}_{1}$ & 1471 & 1451 & 1387 & 1302 & 1230 & 1140 & 1059 & 942 & 806 & 695 \\
\hline $\mathbf{C}_{2}$ & 1491 & 1466 & 1424 & 1380 & 1343 & 1291 & 1221 & 1156 & 1093 & 1031 \\
\hline $\mathrm{C}_{3}$ & 1492 & 1474 & 1458 & 1432 & 1400 & 1359 & 1322 & 1278 & 1239 & 1203 \\
\hline Mean & 1485 & 1464 & 1423 & 1371 & 1324 & 1263 & 1201 & 1125 & 1046 & 695 \\
\hline SEm \pm & 5 & 6 & 3 & 3 & 4 & 5 & 5 & 6 & 6 & 6 \\
\hline CD at $1 \%$ & NS & NS & 12.00 & 12 & 17 & 19 & 18 & 21 & 22 & 21 \\
\hline
\end{tabular}

NS - Non significant; Storage conditions (C); $\mathrm{C}_{1}-$ Ambient storage condition; $\mathrm{C}_{2}$ - Conditioned cold storage $\left(18-20{ }^{\circ} \mathrm{C}, 45-50 \% \mathrm{RH}\right) ; \mathrm{C}_{3}-$ Commercial cold storage condition; (5 to $7^{\circ} 65 \% \mathrm{RH}$ )

Table.8 Effect of storage conditions on electrical conductivity $\left(\mathrm{dSm}^{-1}\right)$ of onion seed during storage

\begin{tabular}{|c|c|c|c|c|c|}
\hline \multirow{2}{*}{ Treatment } & \multicolumn{5}{|c|}{ Storage period in months } \\
\cline { 2 - 6 } & $\mathbf{2}$ & $\mathbf{4}$ & $\mathbf{6}$ & $\mathbf{8}$ & $\mathbf{1 0}$ \\
\hline $\mathbf{C}_{\mathbf{1}}$ & 0.391 & 0.458 & 0.476 & 0.543 & 0.588 \\
\hline $\mathbf{C}_{\mathbf{2}}$ & 0.389 & 0.454 & 0.465 & 0.511 & 0.569 \\
\hline $\mathbf{C}_{\mathbf{3}}$ & 0.388 & 0.452 & 0.462 & 0.514 & 0.566 \\
\hline Mean & 0.389 & 0.454 & 0.468 & 0.523 & 0.574 \\
\hline SEm \pm & 0.0020 & 0.0004 & 0.0005 & 0.0005 & 0.0004 \\
\hline CD at 1 \% & NS & 0.0016 & 0.0018 & 0.0018 & 0.0015 \\
\hline
\end{tabular}

NS - Non significant; Storage conditions (C); $\mathrm{C}_{1}-$ Ambient storage condition; $\mathrm{C}_{2}$ - Conditioned cold storage $\left(18-20{ }^{\circ} \mathrm{C}, 45-50 \% \mathrm{RH}\right) ; \mathrm{C}_{3}-$ Commercial cold storage condition; $\left(5\right.$ to $\left.7{ }^{\circ} 65 \% \mathrm{RH}\right)$

Table.9 Effect of storage conditions on dehydrogenase enzyme activity (OD value) of onion seed during storage

\begin{tabular}{|c|c|c|c|c|c|}
\hline \multirow{2}{*}{ Treatment } & \multicolumn{5}{|c|}{ Storage period in months } \\
\cline { 2 - 6 } & $\mathbf{2}$ & $\mathbf{4}$ & $\mathbf{6}$ & $\mathbf{8}$ & $\mathbf{1 0}$ \\
\hline $\mathbf{C}_{\mathbf{1}}$ & 1.288 & 1.257 & 1.108 & 0.948 & 0.771 \\
\hline $\mathbf{C}_{\mathbf{2}}$ & 1.295 & 1.274 & 1.185 & 1.085 & 0.971 \\
\hline $\mathbf{C}_{\mathbf{3}}$ & 1.298 & 1.289 & 1.254 & 1.187 & 1.104 \\
\hline Mean & 1.294 & 1.273 & 1.182 & 1.073 & 0.949 \\
\hline SEm \pm & 0.0039 & 0.0012 & 0.0010 & 0.0011 & 0.0013 \\
\hline CD at 1 \% & NS & 0.0045 & 0.0040 & 0.0044 & 0.0050 \\
\hline
\end{tabular}

NS - Non significant; Storage conditions (C); $\mathrm{C}_{1}-$ Ambient storage condition; $\mathrm{C}_{2}$ - Conditioned cold storage $\left(18-20{ }^{\circ} \mathrm{C}, 45-50 \% \mathrm{RH}\right) ; \mathrm{C}_{3}-$ Commercial cold storage condition; (5 to $7{ }^{\circ} 65 \% \mathrm{RH}$ ) 
Table.10 Effect of storage conditions on alpha amyalse enzyme activity ( $\mathrm{mm}$ ) of onion seed during storage

\begin{tabular}{|c|c|c|c|c|c|}
\hline \multirow{2}{*}{ Treatment } & \multicolumn{5}{|c|}{ Storage period in months } \\
\cline { 2 - 6 } & $\mathbf{2}$ & $\mathbf{4}$ & $\mathbf{6}$ & $\mathbf{8}$ & $\mathbf{1 0}$ \\
\hline $\mathbf{C}_{\mathbf{1}}$ & 24.00 & 22.02 & 18.25 & 16.31 & 14.38 \\
\hline $\mathbf{C}_{\mathbf{2}}$ & 24.05 & 22.07 & 20.09 & 18.10 & 16.87 \\
\hline $\mathbf{C}_{\mathbf{3}}$ & 24.07 & 23.08 & 21.26 & 19.54 & 18.47 \\
\hline Mean & 24.04 & 22.39 & 19.86 & 17.98 & 16.57 \\
\hline SEm \pm & 0.393 & 0.006 & 0.016 & 0.012 & 0.011 \\
\hline CD at 1 \% & NS & 0.022 & 0.064 & 0.048 & 0.043 \\
\hline
\end{tabular}

NS - Non significant; Storage conditions (C); $\mathrm{C}_{1}-$ Ambient storage condition; $\mathrm{C}_{2}-$ Conditioned cold storage

$\left(18-20{ }^{\circ} \mathrm{C}, 45-50 \% \mathrm{RH}\right) ; \mathrm{C}_{3}-$ Commercial cold storage condition; (5 to $7{ }^{\circ} 65 \% \mathrm{RH}$ )

Seedling vigour was significantly influenced by the storage conditions for all storage periods Table 7. Seedling vigour index was decreased with the increase of storage period. After one month of storage highest seedling vigour index (1492) was observed in commercial cold storage $\left(\mathrm{C}_{3}\right)$ which was followed by conditioned cold storage $\left(\mathrm{C}_{2}\right)$ (1491) and lowest was noticed in ambient condition $\left(\mathrm{C}_{1}\right)(1471)$. As the storage period advances the vigour of seed declines due to catabolic activity going on in the seed and thus the seed though viable, yet fails to emerge. Decline in seed vigour depends on storage condition that is temperature, relative humidity and seed moisture contents. High temperature, relative humidity and moisture in the storage environment appear to be principle factors involved in deterioration of seed quality however at the end of ten months of storage period, significantly highest seedling vigour index (1203) was recorded in commercial cold storage $\left(\mathrm{C}_{3}\right)$, which was followed by conditioned cold storage $\left(\mathrm{C}_{2}\right)$ (1031) and significantly lowest seedling vigour index was recorded in ambient condition $\left(\mathrm{C}_{1}\right)$ (695). The increase in seedling vigour index in controlled condition can be attributed to increased seed germination and seedling length. These findings are similar with findings of Balesevic Tubic et al., (2010) in oilseed crops, Basavegowda et al., (2013) in chickpea, and Venge et al., (2016) in soybean, Patel et al., (2017) in onion and Meena et al., (2017) in groundnut.
The electrical conductivity $\left(\mathrm{dSm}^{-1}\right)$ of onion seeds differed significantly except first and second month of storage period between the storage conditions at all the stages of storage (Table 8). However, electrical conductivity was lowest in seeds stored in commercial cold storage $\left(\mathrm{C}_{3}\right)\left(0.389 \mathrm{dSm}^{-1}\right)$ which was followed by conditioned cold storage $\left(\mathrm{C}_{2}\right)\left(0.389 \mathrm{dSm}^{-1}\right)$ and highest electrical conductivity $\left(\mathrm{C}_{1}\right)(0.391$ $\mathrm{dSm}^{-1}$ ) was observed in ambient condition after second month of storage period. The lower electrical conductivity of seed leachate in cold storage was mainly governed by lower cell wall permeability may be due to lower temperature in cold storage $\left(5-7{ }^{\circ} \mathrm{C}\right)$ and might have helped in controlling lower respiration rate and metabolic activity and maintenance of vigour during storage. Similarly the seeds preserved in commercial cold storage have recorded lower electrical conductivity $(0.566$ $\mathrm{dSm}^{-1}$ ) at the end of ten months of storage, which was followed by conditioned cold storage $\left(\mathrm{C}_{2}\right)\left(0.569 \mathrm{dSm}^{-1}\right)$ and significantly highest electrical conductivity was recorded in ambient condition $\left(\mathrm{C}_{1}\right)\left(0.588 \mathrm{dSm}^{-1}\right)$. These findings are supported by results of similar studies of Ellis et al., (1991) in onion, Basave Gowda et al., (2013) in chickpea, Meena et al., (2017) in groundnut and Meena et al., (2017) in cotton.

The enzyme activity is a good stable metabolic marker to estimate the degree of vigour in seeds (Saxena et al., 1987) and have positive association with vigour and viability of seeds 
(Halder and Gupta, 1982 and Rudrapal and Basu, 1982) (Table 9 and10). The highest total dehydrogenase activity and alpha amylase activity (1.298 OD value and $24.07 \mathrm{~mm}$ ) was recorded in commercial cold storage $\left(\mathrm{C}_{3}\right)$ which was followed by conditioned cold storage $\left(\mathrm{C}_{2}\right)$ (1.295 OD value and $\left.24.05 \mathrm{~mm}\right)$ and lowest was noticed in ambient condition (1.288 OD value and $24.00 \mathrm{~mm}$ ) after two months of storage. The enzyme activity decreased with the advancement in storage period might be due to the inability of the seed tissues to reduce tetrazolium chloride to insoluble formazan. At the end of tenth months of storage period, significantly highest dehydrogenase activity and alpha amylase activity (1.104 OD value and $18.47 \mathrm{~mm}$ ) was recorded in $\mathrm{C}_{3}$, which was followed by $\mathrm{C}_{2}$ (0.971 OD value and $16.87 \mathrm{~mm})$ and significantly lowest was recorded in $C_{1}(0.771$ OD value and $14.38 \mathrm{~mm}$ ). These results were in accordance with the findings of Pallavi et al., (2003) in sunflower.

In conclusion, it has been found from the present investigation that, the shelf life of onion seeds can be extended up to ten months with above minimum seed certification standard by storing in commercial cold storage maintained higher germination, speed of germination, shoot length, root length, seedling vigour index, dehydrogenase activity, alpha amylase and lower moisture content and electric conductivity compared to ambient condition.

\section{References}

Abdul-Baki, A. A. and Anderson, J. D., 1973, Physiological and biochemical deterioration of seeds. In: Seed Biology. Ed. T.T. Kozlowski. Academic Press, New York, pp: 283-315.

Akbari, G. E., Ghassemi, P. E. A., Najafabadi, M. and Shahverdi, M., 2004, Studying physiological seed quality on soybean. Agric. J., 1(6): 9-18.
Alhamdan, A. M., Alsadon, A. A., Khalil, S.O., Mahmoud, A.W., Nagar, M.E. and Ibrahim, A.A., 2011, Influence of storage conditions on seed quality and longevity of four vegetable crops. American-Eurasian $J$. Agric. and Envi. Sci., 11(3): 353-359.

Balesevic , T. S., Tatic, M., Dordevic, V., Nilolic, Z. and Dukic, V., 2010, Seed viability of oil crops depending on storage conditions. Helia., 33(52): 153-160.

Basavegowda, Gururaj, S. and Arunkumar, H., 2013, Effect of commercial cold storage conditions and packaging materials on seed quality of chickpea (Cicer Arietinum.L). Global. J. Sci. Fro. Res., 13(2): 23-27.

Basavegowda, Gururaj, S. and Arunkumar, H., 2013, Effect of commercial cold storage conditions and packaging materials on seed quality of chickpea (Cicer Arietinum.L). Global. J. Sci. Fro. Res., 13(2): 23-27.

Delouche, J. C. and Baskin, C. C., 1973, Accelerated ageing techniques for predicting the relative storability of seed lots. Seed Sci. and Technol., 1: 427-452.

Demir, I., Ozden, E., Kara, F., Hassanzadeh, H. and Mavi, K., 2016, Effect of ambient storage temperature and seed moisture content on seed longevity of lettuce. American. J. Expt. Agric., 12(3): 1-5.

Demir, I., Ozden, E., Kara, F., Hassanzadeh, H. and Mavi, K., 2016, Effect of ambient storage temperature and seed moisture content on seed longevity of lettuce. American. J. Expt. Agric., 12(3): 1-5.

Devanal M., Basevegowda, Vasudevan, S. N., Shekargoud, I. and Gururajsunkad, 2012, Effect of storage conditions and packing materials on seed quality of sunflower (Helianthus annus L.). National seed congress, Chandigarh, pp.174-175.

Halder, S. and Gupta, K., 1982, On the mechanism of sunflower seed deterioration under low and high relative humidity. Seed Sci. and Tech., 10: 267-270.

Hanna, D., Magdalena, J., Dorota, S., Izabela, S. and Agnieszka, R., 2013, Germination, vigour and health of primed (Allium cepa L.) seeds after storage. Acta Sci. Pol., 12(4): 43-58.

Harrington, J. F., 1960, Thumb rules of drying seed. Crops and Soils, 13: 16-17 
ISTA, 1999, International Rules of Seed Testing. Seed Sci. and Technol., 27: 25-30.

Kandil, A. A., Sharief, A. E. and Sheteiwy, M. S., 2013, Seedling parameters of soybean cultivars as influenced with seed storage periods, conditions and materials. Int. J. Agric. Sci., 5(1): 330-336.

Kavitha, S., Menaka, C. and Ananthi, M., 2017, Deterioration in sesame (Sesamum indicum L.) seeds under natural and accelerated ageing. Int. J. Chemical Studies, 5(4): 11411146.

Khalequzzaman, K. M., Rashid, M. M., Hasan, M. A. and Reza, M. A., 2012, Effect of storage containers and storage periods on the seed quality of french bean (Phaseolu vulgaris L.). Bang. J. Agril. Res., 37(2): 195-205.

Kittock, D. L. and Law, A. G., 1968, Relationship of seedling vigour, respiration and tetrazolium chloride reduction by germination of wheat seeds. Agron. J., 60: 286-288.

Meena, M. K., Chetti, M. B. and Nawalagatti, C. M., 2017a, Influence of different packaging materials and storage conditions on the seed quality parameters of groundnut (Arachis hypogaea L.), Int. J. Pure App. Biosci., 5(1): 933-941.

Meena, M. K., Chetti, M. B. and Nawalagatti, C. M., 2017b, Influence of vacuum packaging and storage conditions on the seed quality of cotton (Gossypium spp.), Int. J. Pure App. Biosci, 5(1): 789-797.

Milosevic, M. M., Vujakovic, D. and Karagic., 2010, Vigour tests as indicators of seed viability. Genetika, 42(1): 103-118.

Na, Y. W., Shim, S. I., Chung, J. S., Rho, I. R. and Kim, S. H., 2013, The effect of pellet materials and storage conditions on the viability and water contents of pelleted

\section{How to cite this article:}

Geetanjali, C., I.M. Sangeeta, S.M. Prashant, Basavegowda and Beladhadi, R.V. 2019. Effect of Storage Conditions on Seed Longevity of Onion (Allium cepa L.). Int.J.Curr.Microbiol.App.Sci. 8(02): 1897-1905. doi: https://doi.org/10.20546/ijcmas.2019.802.221 seeds in sesame (Sesamum indicum L.). J. Korean Soc. Int. Agric., 25: 417-422.

Pallavi, M., Sudheer, K. S., Kuldeep, S. D. and Vishnuvardhan, R. A., 2003, Effect of seed ageing on physiological, biochemical and field attributes in sunflower (Helianthus annuus L.) cv. Morden. Seed Res, 31: 4648.

Patel, J. B., Babariya, C. A., Jyoti, S., Ribadiya, K. H. and Bhatiya, V. J., 2017, Effect of storage conditions, packing materials and seed treatments on viability and seedling vigour of onion (Allium cepa L.) seeds. J. Appl. and Nat. Sci., 9(2): 1054-1067.

Rudrapal, A. B. and Basu, R. N., 1982, Lipid peroxidation and membrane damage in deteriorating wheat and mustard seeds. Indian J. Expt. Bio., 20: 465-470.

Saxena, O. P, Singh, G., Pakeeraiah, T. and Pandey, N., 1987, Seed deterioration studies in some vegetable seeds. Acta. Hort., 215: $39-44$.

Simpson, G. M. and Naylar, J. M., 1962, Dormancy studies in seeds of Avena fatuva and relationship between maltase, amylases and gibberellins. Can. J. Bot., 40: 19591673.

Suma, A., Sreenivasan, K., Singh, A. K. and Radhamani, J., 2013, Role of relative humidity in processing and storage of seeds and assessment of variability in storage behaviour in Brassica spp. and Eruca sativa. The Sci. World. J., 9: 1-5.

Venge, T., Ikyeleve, F. and Oko, J. O., 2016, Effect of packaging materials and storage condition on soybean germination and seedling vigour in Makurdi. Res. J. Seed Sci., 9(1): 1-4. 